\title{
Leukocytosis at Diagnosis in Patients with Essential Thrombocythemia Is a Risk Factor for Transformation into Myelofibrosis
}

\author{
Irene Bertozzi, Fabiana Tezza, Emanuela Bonamigo, Fabrizio Fabris, Maria Luigia Randi* \\ Department of Medicine-DIMED, University of Padua, Padua, Italy. \\ Email: ${ }^{*}$ marialuigia.randi@unipd.it
}

Received July $17^{\text {th }}, 2012$; revised August 19 ${ }^{\text {th }}, 2012$; accepted August $27^{\text {th }}, 2012$

\begin{abstract}
Myelofibrosis (MF) represents the major long-term complication of essential thrombocythemia (ET). There is evidence that leukocytosis at diagnosis is associated with poorer survival in patients with ET. In this study, we retrospectively evaluated 143 patients with ET, diagnosed in agreement with WHO criteria, followed in a single centre over 10 years. Nine of them transformed into MF (post-essential thrombocythemia-myelofibrosis PET-MF). We compared PET-MF data at diagnosis with that of the remaining 134 patients (ET-1) and with a selected sub-group of ET-1 (ET-2, 19 pats) sex, age and follow-up duration matched to PET-MF. The PET-MF evolution rate was 4.6 per 1000 person-years; white blood cells count (WBC) count, haemoglobin levels and hematocrit were higher in PET-MF than in ET-1 $(P=0.01)$ while only WBC was higher than in ET-2 $(P=0.01)$. With multivariate analysis, only WBC count retained its significance. Our study highlights the prognostic relevance of leukocytosis on myelofibrotic transformation of ET.
\end{abstract}

Keywords: Essential Thrombocythemia; Leukocytosis; Post-Thrombocythemia Myelofibrosis; Myeloproliferative Neoplasms

\section{Introduction}

Among the classical myeloproliferative neoplasms (MPN), essential thrombocythemia (ET) has the most favourable prognosis in terms of survival and myelofibrosis or leukemic transformation: ET is considered usually not to reduce the patient's life expectancy, with median survival ranging from 13 to 22.3 years [1]. However, a recent paper [2] showed an $83 \%$ survival at 10 years and $47 \%$ at 20 years after diagnosis that resulted significantly lower than the expected survival of an age and sex matched control population. Interestingly, leukocytosis at diagnosis was associated with poorer survival in ET as well as in polycythemia vera (PV) [3].

The transition to MF represents the major long-term complication of ET with an estimated risk of about $5 \%$ at 10 years [4] and the transition to MF is considered a facilitating condition to the development of acute leukemia (AL). Leukocytosis has been published to be an adverse prognostic factor for leukemic transformation [5] and significant [6] or near-significant [7] associations in PV in this regard have previously been communicated as well. In contrast, no meaningful difference between the presence of $J A K 2 \mathrm{~V} 617 \mathrm{~F}$ mutation and the progression to

\footnotetext{
${ }^{*}$ Corresponding author.
}

PET-MF has been observed [8], even if in a large study [9] a greater risk of developing large splenomegaly and leukemia was found in JAK2V617F mutated PMF patients and the accumulation of mutant $\mathrm{V} 617 \mathrm{~F}$ alleles has been reported to be possibly associated to evolution of PV to MF [10].

In the present study, we evaluated some easy data registered at diagnosis of ET to define if leukocytosis is "per se" a risk factor for MF progression in these patients.

\section{Methods}

We evaluated 143 consecutive patients (106 females and 37 males) affected by ET followed in our Department. The study was conducted in accordance with institutional guidelines established for retrospective studies, and has been approved from the Ethical Committee of Padua General Hospital.

The World Health Organization (WHO) criteria were used to diagnose ET [11]. Earlier cases diagnosed according to the polycythemia vera study group criteria were re-reviewed and included in the current study only if they also fulfilled the WHO diagnostic criteria. The ET transformation into PET-MF was defined in agreement with the criteria of the International Working Group for 
myelofibrosis [12]. Hydroxyurea as cytoreductive treatment in high risk patients and low dose aspirin as primary and secondary thrombotic prevention were used.

The patients were divided in those who progressed to myelofibrosis (PET-MF, 9 patients, median follow up at transformation 11.7 years) and those who did not (ET-1, 134 patients). Considering that ET-1 patients had very wide follow-up duration (range 2 - 27 years), we selected within ET-1 group, 19 patients (ET-2) who were comparable for sex, age and follow-up duration to PET-MF patients. The main characteristics of the groups are summarized in Table 1.

All analyses were based on the laboratory parameters obtained at time of diagnosis. All $P$-values were twotailed and statistical significance was set at the level of $P$ $<0.05$. Comparison between categorical variables was performed by chi-squared statistics. The comparison between means was performed with one-way ANOVA and the threshold of WBC number has been defined with ROC curve. Cox proportional hazards model was utilised to determine the impact of various clinical and laboratory variables on overall, MF-free survival. Overall survival curves were constructed by Kaplan-Meier method taking the interval from the date of diagnosis.

\section{Results}

At the end of the study, 132 out of our 143 patients were alive, while 10 ET-1 (2 acute leukemia) and 1 PET-MF died (no leukemic transformation).

The PET-MF incidence was 4.6 per 1000 person-years, with a 10 -year risk evolution rate of $3 \%$. In univariate analysis, WBC count, haemoglobin level and hematocrit at diagnosis were statistically higher in PET-MF than in ET-1. This result was confirmed only for the WBC count at diagnosis when PET-MF was compared with ET-2 (Table 2).

The threshold WBC count significant for PET-MF was identified at $10.2 \times 10^{9} / \mathrm{L}$ (sensibility $83 \%$, specificity $81 \%$ ) comparing with ET-1 and at $10 \times 10^{9} / \mathrm{L}$ (sensibility $87.5 \%$, specificity $88.2 \%$ ) comparing with ET-2. Platelet counts were statistically similar in all patients.

Table 1. Characteristics in gender, follow-up duration and JAK2 mutation of our patients. The number of reviewed bone marrow (BOM) histology in each group is reported as well. No significant difference in age at diagnosis, follow-up duration and frequency of JAK2V617F mutation was observed between the different groups.

\begin{tabular}{cccc}
\hline & PET-MF & ET-1 & ET-2 \\
\hline Gender (M/F) & $4 / 5$ & $32 / 102$ & $9 / 10$ \\
Mean age at diagnosis (years) & $53.4 \pm 17.7$ & $51.0 \pm 17.3$ & $54.3 \pm 15.0$ \\
Median total follow up (years) & 13.2 & 10.38 & 12.9 \\
JAK2, V617F/WT & $6 / 3$ & $67 / 42$ & $13 / 6$ \\
Reviewed BOM & 9 & 98 & 15 \\
\hline
\end{tabular}

Table 2. Comparison of clinical and laboratory characteristics of our patients divided in subgroups as observed at the time of diagnosis of ET. PET-MF = patients transformed into MF, ET-1 = all patients who did not transformed, ET-2 = patients not transformed age sex and follow-up duration matched to the group of PET-MF.

\begin{tabular}{|c|c|c|c|c|c|}
\hline & PET-MF & ET-1 & $P$ & ET-2 & $P$ \\
\hline $\mathrm{WBC} \times 10^{9} / \mathrm{L}$ & $12 \pm 3.6$ & $8.5 \pm 2.6$ & $<0.01$ & $7.8 \pm 2.4$ & $<0.01$ \\
\hline Hemoglobin $\mathrm{g} / \mathrm{dL}$ & $150 \pm 13$ & $133 \pm 15$ & $<0.01$ & $137 \pm 13$ & n.s. \\
\hline Hematocrit \% & $46.8 \pm 4.3$ & $41 \pm 4.5$ & $<0.01$ & $43 \pm 3.4$ & n.s. \\
\hline Platelets $\times 10^{9} / \mathrm{L}$ & $804 \pm 396$ & $777 \pm 278$ & n.s. & $690 \pm 148$ & n.s. \\
\hline Anemia yes/no ${ }^{*}$ & $1 / 8$ & $19 / 115$ & n.s. & $1 / 18$ & n.s. \\
\hline Male/Females & $4 / 5$ & $32 / 102$ & n.s. & $9 / 10$ & n.s. \\
\hline Age $>60$ years yes $/$ no & $5 / 4$ & $50 / 84$ & n.s. & $9 / 10$ & n.s. \\
\hline Splenomegaly yes/no* & $5 / 4$ & $44 / 90$ & n.s. & $3 / 16$ & 0.02 \\
\hline Thrombosis & $5 / 4$ & $41 / 93$ & n.s. & $3 / 16$ & 0.02 \\
\hline Hydroxyurea yes/no & $9 / 0$ & $81 / 53$ & n.s. & $12 / 7$ & n.s. \\
\hline
\end{tabular}

\footnotetext{
${ }^{*}$ Anemia $=<125 \mathrm{~g} / \mathrm{L}$ in females and $<133 \mathrm{~g} / \mathrm{L}$ in males; ${ }^{* *}$ Splenomegaly $=$ palpable spleen $>1 \mathrm{~cm}$ or enlarged at morphologic ultrasound.
} 
The age at diagnosis, the presence of anemia and the use of hydroxyurea were comparable in the 3 different groups of patients. The presence of splenomegaly and the occurrence of thrombosis were significantly more common in PET-MF than in ET-2 but not when compared to ET-1. The use of aspirin was more frequent in PET-MF than in ET-2. In multivariate analysis, the Cox regression was significant only for WBC values both comparing ET-1 vs PET-MF and ET-2 vs PET-MF. In univariate analysis, MF-free survival was significantly associated with leukocytosis using the cut-off level of $\geq 10 \times 10^{9} / \mathrm{L}$ $(P=0.001)$ (Figure 1).

\section{Discussion}

Thrombo-hemorrhagic events and transformation into acute leukemia or myelofibrosis are the main causes of morbidity and mortality in patients with ET [13].

After some sporadic cases reported of ET transforming in MF, Murphy in 1997 [14] observed 3 cases of PET-MF out of 50 cases after a median time of 6 years; Cervantes et al. [15] found a probability of developing MF of $8 \%$ at 10 years and $15 \%$ at 15 years. At present, the risk of myelofibrosis in ET patients is considered to occur at least 10 years after diagnosis [16] being of about $5 \%$ [4]. Indeed, the transition to MF represents the major long-term complication of ET.

In the recent years, a great attention has been devoted to the significance of WBC count in patients affected by myeloproliferative neoplasms (MPN): high WBC at diagnosis seems to be a risk for thrombotic complications in PV [17] and in ET [18].

Our study confirms that MF transformation occurs after a minimum of 10 years after ET diagnosis and shows that an high number of WBC at diagnosis is a relevant marker to predict MF progression in patients with ET. In contrast,

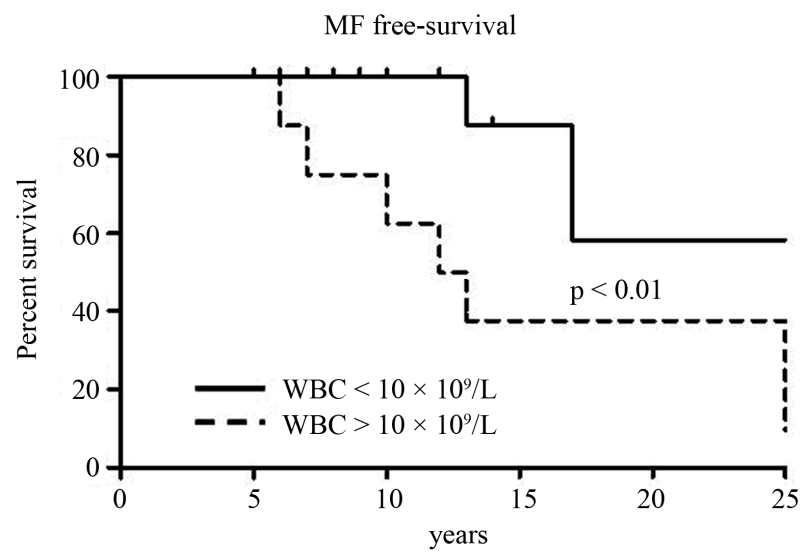

Figure 1. Myelofibrosis-free survival (MF-free survival) in 143 patients with essential thrombocythemia stratified on the basis of white blood cells count (WBC) count at diagnosis. gender, advanced age at diagnosis, the presence of anemia, or platelet number are not significant in contrast to the observation of other Authors [19,20]. It is possible that this finding may be justified by the small number of cases of PET-MF we have found. Notably, in agreement with other authors [20] we did not find any correlation between the use of cytotoxic agents and post-thrombocythemia myelofibrosis suggesting that myelofibrosis, although rare, is a natural evolution of ET $[10,15]$.

In 311 retrospective studied ET patients in France, $\mathrm{WBC}>11 \times 10^{9} / \mathrm{L}$ at the time of diagnosis was associated with poorer survival [2] and other Authors [3,5] found that WBC $>15 \times 10^{9} / \mathrm{L}$ were associated with a worse prognosis. Leukocytosis has been identified as an adverse prognostic factor for leukemic transformation both in PV and ET [3,6,7] and in some cases leukemic evolution is preceded by a period of myelofibrotic phenotype; however, at the moment, none of our PET-MF developed leukemia but this thought stimulate to control more strictly PET-MF patients. Finally, because JAK2 allele burden has been shown to increase during the follow-up of patients developing MF [10] our data suggest to monitor allele burden mainly in patients with ET and WBC number $>10 \times 10^{9} / \mathrm{L}$ at diagnosis.

Recently, the different prognosis of ET and early/prefibrotic PMF has been underlined and some risk factors included high leukocyte count have been proposed as markers of occult early/prefibrotic PMF explaining their association with inferior survival or higher risk of disease progression [21]. The current study highlighted the prognostic relevance of leukocytosis on myelofibrotic transformation of ET [22], as it has been reported for PV [3]. Our findings with regard to the survival disadvantage linked to leukocytosis in ET [5,22] are consistent with a similar scenario seen in the context of the other two classic BCR-ABL-negative MPNs including PV [6] and primary myelofibrosis.

Regardless, prospective controlled studies are necessary to determine whether or not early cytoreductive therapy in MPNs would favourably influence the adverse prognosis associated with leukocytosis.

\section{Acknowledgements}

This study was supported in part by the Italian MURST grants.

\section{REFERENCES}

[1] C. Rozman, E. Feliu, M. Giralt, D. Rubio and M. T. Cortés, "Life Expectancy of Patients with Chronic Nonleukemic Myeloproliferative Disorders," Cancer, Vol. 67, No. 10, 1991, pp. 2656-2663. doi:10.1002/1097-0142(19910515)67:10<2658::AID-CN 


\section{CR2820671042>3.0.CO;2-C}

[2] F. Girodon, F. Dutrillaux, J. Broséus, M. Mounier, V. Goussot, P. Bardonnaud, et al., "Leukocytosis Is Associated with Poor Survival But Not with Increased Risk of Thrombosis in Essential Thrombocythemia: A Population-Based Study of 311 Patients," Leukemia, Vol. 24, No. 4, 2010, pp. 900-903. doi:10.1038/leu.2010.5

[3] N. Gangat, J. Strand, C.-Y. Li, W. T. Wu, A. Pardanani and A. Tefferi, "Leukocytosis in Polycythemia Vera Predicts Both Inferior Survival and Leukemic Transformation," British Journal of Haematology, Vol. 138, No. 3, 2007, pp. 354-358.

doi:10.1111/j.1365-2141.2007.06674.x

[4] F. Palandri, L. Catani, N. Testoni, E. Ottaviani, N. Polverelli, M. Fiacchini, et al., "Long-Term Follow-Up of 386 Consecutive Patients with Essential Thrombocythemia: Safety of Cytoreductive Therapy," American Journal of Hematology, Vol. 84, No. 4, 2009, pp. 215-220. doi:10.1002/ajh.21360

[5] N. Gangat, A. P. Wolanskyj, R. F. McClure, C.-Y. Li, S. Schwager, W. Wu and A. Tefferi, "Risk Stratification for Survival and Leukemic Transformation in Essential Thrombocythemia: A Single Institutional Study of 605 Patients," Leukemia, Vol. 21, No. 2, 2007, pp. 270-276. doi:10.1038/sj.leu.2404500

[6] J. J. Kiladjian, C. Gardin, M. Renoux, F. Bruno and J. F. Bernard, "Long-Term Outcomes of Polycythemia Vera Patients Treated with Pipobroman as Initial Therapy," The Hematology Journal, Vol. 4, 2003, pp. 198-207. doi:10.1038/sj.thj.6200250

[7] G. Finazzi, V. Caruso, R. Marchioli, G. Capnist, T. Chisesi, C. Finelli, et al., "Acute Leukemia in Polycythemia Vera: An Analysis of 1638 Patients Enrolled in a Prospective Observational Study," Blood, Vol. 105, No. 7, 2005, pp. 2664-2670. doi:10.1182/blood-2004-09-3426

[8] P. Guglielmelli, G. Barosi, L. Pieri, E. Antonioli, A. Bosi and A. M. Vannucchi, "JAK2V617F Mutational Status and Allele Burden Have Little Influence on Clinical Phenotype and Prognosis in Patients with Post-Polycythemia Vera and Post-Essential Thrombocythemia Myelofibrosis," Haematologica, Vol. 94, No. 1, 2009, pp. 144-146. doi:10.3324/haematol.13721

[9] G. Barosi, G. Bergamaschi, M. Marchetti, A. M. Vannucchi, P. Guglielmelli, E. Antonioli, et al., "JAK2V617F Mutational Status Predicts Progression to Large Splenomegaly and Leukemic Transformation in Primary Myelofibrosis," Blood, Vol. 110, No. 12, 2007, pp. 4030-4036. doi:10.1182/blood-2007-07-099184

[10] F. Passamonti, E. Rumi, D. Pietra, C. Elena, E. Boveri, E. Roncoroni, et al., "A Prospective Study of 338 Patients with Polycythemia Vera: The Impact of JAK2(V617F) Allele Burden and Leukocytosis on Fibrotic or Leukemic Disease Transformation and Vascular Complications," Leukemia, Vol. 24, No. 9, 2010, pp. 1574-1579. doi:10.1038/leu.2010.148

[11] J. W. Vardiman, J. Thiele, D. A. Arber, R. D. Brunning, M. J. Borowitz, A. Porwit, et al., "The 2008 Revision of the WHO Classification of Myeloid Neoplasms and
Acute Leukemia: Rationale and Important Changes," Blood, Vol. 114, No. 5, 2009, pp. 937-951. doi:10.1182/blood-2009-03-209262

[12] G. Barosi, R. Mesa, J. Thiele, F. Cervantes, P. J. Campbell, S. Verstovsek, et al., "Proposed Criteria for the Diagnosis of Post-Polycythemia Vera and Post-Essential Thrombocythemia Myelofibrosis: A Consensus Statement from the International Working Group for Myelofibrosis Research and Treatment," Leukemia, Vol. 22, No. 2, 2008, pp. 437-438. doi:10.1038/sj.leu.2404914

[13] M. A. Elliott, S.-Y. Yoon, P. Kao, C.-Y. Li and A. Tefferi, "Simultaneous Measurement of Serum Thrombopoietin and Expression of Megakaryocyte c-Mpl with Clinical and Laboratory Correlates for Myelofibrosis with Myeloid Metaplasia," European Journal of Haematology, Vol. 68, No. 3, 2002, pp. 175-179. doi:10.1034/j.1600-0609.2002.01534.x

[14] S. Murphy, P. Peterson, H. Iland and J. Laszlo, "Experience of the Polycythemia Vera Study Group with Essential Thrombocythemia: A Final Report on Diagnostic Criteria, Survival and Leukemic Transformation by Treatment," Seminars in Hematology, Vol. 34, No. 1, 1997, pp. 29-39.

[15] F. Cervantes, A. Alvarez-Larrán, C. Talarn, M. Gómez and E. Montessart, "Myelofibrosis with Myeloid Metaplasia Following Essential Thrombocythemia: Actuarial Probability, Presenting Characteristics and Evolution in a Series of 195 Patients," British Journal of Haematology, Vol. 118, No. 3, 2002, pp. 786-790. doi:10.1046/j.1365-2141.2002.03688.x

[16] F. Passamonti, E. Rumi, E. Pungolino, L. Malabarba, P. Bertazzoni, M. Valentini, et al., "Life Expectancy and Prognostic Factors for Survival in Patients with Polycythemia Vera and Essential Thrombocythemia," The American Journal of Medicine, Vol. 117, No. 10, 2004, pp. 755-761. doi:10.1016/j.amjmed.2004.06.032

[17] R. Landolfi, L. Di Gennaro, T. Barbui, V. De Stefano, G. Finazzi, R. M. Marfisi, et al., "Leukocytosis as a Major Thrombotic Risk Factor in Patients with Polycythemia Vera," Blood, Vol. 109, No. 6, 2007, pp. 2446-2452. doi:10.1182/blood-2006-08-042515

[18] A. Carobbio, E. Antonioli, P. Guglielmelli, A. M. Vannucchi, F. Delaini, V. Guerini, et al., "Leukocytosis and Risk Stratification Assessment in Essential Thrombocythemia," Journal of Clinical Oncology, Vol. 26, No. 16, 2008, pp. 2732-2736. doi:10.1200/JCO.2007.15.3569

[19] F. Passamonti, E. Rumi, L. Arcaini, E. Boveri, C. Elena, D. Pietra, et al., "Prognostic Factors for Thrombosis, Myelofibrosis and Leukemia in Essential Thrombocythemia: A Study of 605 Patients," Haematologica, Vol. 93, No. 11, 2008, pp. 1645-1651. doi:10.3324/haematol.13346

[20] A. Tefferi, N. Gangat, A. P. Wolanskyj, S. Schwager, A. Pardanani, T. L. Lasho, et al., " $20+$ yr without Leukemia or Fibrotic Transformation in Essential Thrombocythemia or Polycythemia Vera: Predicts at Diagnosis," European Journal of Haematology, Vol. 80, No. 5, 2008, pp. 386390. doi:10.1111/j.1600-0609.2008.01038.x 
[21] T. Barbui, J. Thiele, F. Passamonti, E. Rumi, E. Boveri, M. Ruggeri, et al., "Survival and Disease Progression in Essential Thrombocythemia Are Significantly Influenced by Accurate Morphologic Diagnosis: An International Study," Journal of Clinical Oncology, Vol. 29, No. 23, 2011, pp. 3179-3184. doi:10.1200/JCO.2010.34.5298
[22] A. P. Wolanskyj, S. M. Schwager, R. F. McClure, D. R. Larson and A. Tefferi, "Essential Thrombocythemia beyond the First Decade: Life Expectancy, Long-Term Complication Rates, and Prognostic Factors," Mayo Clinic Proceedings, Vol. 81, No. 2, 2006, pp. 159-166. doi: $10.4065 / 81.2 .159$ 